\title{
El espacio esperas-ocupación como herramienta de monitorización de la calidad del servicio prestado en terminales de contenedores
}

\author{
José Aguilar Herrando
}

Catedrático de explotación de puertos, Universitat Politècnica de València, España

\section{RESUMEN}

En la presente ponencia se desarrollan las bases habituales del cálculo de la capacidad de terminales portuarias de contenedores, haciendo énfasis en la debilidad con la que habitualmente nos encontramos de tener que aplicar valores obtenidos de la teoría de colas, cuando ya sabemos que modelaciones que contemplan variabilidad de esloras y asignación de atraques dan resultados diferentes. Dado que a menudo incluso modelados que contemplen estos casos resultan insuficientes, se concluye la necesidad de los responsables de los puertos asuman su obligación de obtener datos robustos de algunos datos de explotación que nos permitan caracterizar la relación entra la ocupación de los muelles y las colas que se producen.

\section{INTRODUCCIÓN. LA CAPACIDAD DEL ATRAQUE DE UNA TERMINAL PORTUARIA COMO TRÁFICO LÍMITE DEL SERVICIO}

Siguiendo a De Monie (1988), a la hora de hablar de rendimiento portuario, el Tráfico es una de las medidas de la Producción; siendo ésta alguna de las medidas de los volúmenes manipulados por la terminal. Más en concreto, el Tráfico expresa la cantidad neta de mercancía que pasa por la terminal, o por un subsistema de la misma, en un periodo de tiempo. En nuestro caso nos referiremos exclusivamente al subsistema de carga y de descarga, es decir al atraque. Existe otro concepto que De Monie denominó Throughput (que podríamos denominar Transferencia), y que se refiere a la cantidad bruta de mercancía movida, incluyendo así movimientos complementarios (remociones en el buque y tapas de escotilla).

El tráfico del atraque evoluciona en el tiempo, con la demanda que las navieras realizan. Este valor, y todos los demás parámetros de la terminal, evolucionan en el tiempo, de suerte que surge el interés de valorar cuál es el valor límite que nuestro subsistema puede admitir, habitualmente en 1 año. A este tráfico límite le llamamos Capacidad. Entendida pues la Capacidad como un Tráfico límite, es fundamental definir los límites en los que debemos trabajar.

Aparecen así los diferentes conceptos de Capacidad, ligados a la forma de definir estos límites, que tan bien expuso Rodríguez (1977). Estos límites son situaciones idealizadas, por 
lo que la Capacidad es siempre el resultado de una idealización, cuyo valor se estima indirectamente a partir de parámetros y formulaciones, con base a una modelación de la realidad; mientras que el Tráfico es un concepto susceptible de ser medido directamente.

A la hora de calcular la Capacidad nos solemos encontrar en una de las dos situaciones siguientes:

- Dimensionamiento: es el momento del proyecto de la terminal, situación en la que no disponemos de datos reales sobre los parámetros de la instalación, que los debemos pues suponer.

- Comprobación: momento en el que ya disponemos de datos de la explotación, y deseamos conocer los límites de ésta. En esta ponencia asumiremos que estamos en esta situación

Para poder formularla, mediante un simple desarrollo podemos expresar:

- Tráfico $T$ (en uds. de carga/año):

$$
T=\lambda \cdot H \cdot q
$$

- Productividad $\mathrm{P}$ del atraque (en uds. de carga/hora):

$$
P=\frac{q}{t_{S}}
$$

La frecuencia del servicio de la terminal - no la de 1 atraque- $\mu$ (en buques/hora):

$$
\mu=\frac{N}{t_{S}}
$$

.. siendo:

- $\lambda$ : la frecuencia de llegada de buques a toda la terminal (en buques/hora),

- $H$ : el número de horas al año que trabaja la terminal (en horas),

- $q$ : el cargamento medio,

- $t_{S}$ : el tiempo medio de operación de un atraque,

- $\quad N$ : es el número de atraques.

Y con todo ello:

$$
T=\frac{\lambda}{\mu} \cdot N \cdot P \cdot H
$$

La relación $\frac{\lambda}{\mu}$ representa la carga de trabajo de la terminal respecto de la máxima (buques que llegan en relación con los que los que son atendidos); se la suele denominar tasa de ocupación; y la representaremos por $\rho$.

Si (4) formula el valor de $T$, y la Capacidad $C$ es un tráfico límite, se la puede expresar como:

$$
C=\rho_{\text {lim }} \cdot N \cdot P_{\text {lim }} \cdot H_{\text {lim }}
$$

En general $H_{\text {lim }}$ es $H$. Respecto de $P_{\text {lim }}$ se podía abrir un punto de discusión sobre si se toma 
el valor actual $P$; o bien uno futuro, con motivo por ejemplo de estimar el efecto de una mejora en el sistema de grúas. En lo que sigue supondremos que deseamos estimar el límite de la situación actual.

Por último, el valor $\rho_{\text {lim }}$ representa la ocupación máxima que puede admitir el atraque de nuestra terminal. Un resultado inicial trivial nos llevaría a proponer $\rho_{\text {lim }}=1$, pero es bien conocido que en un sistema de llegadas aleatorias ese valor es inalcanzable pues produce colas de longitud infinita.

\section{LA CALIDAD DEL SERVICIO COMO LÍMITE DE LA OCUPACIÓN}

Como ya indicamos en Monfort et alt (2011), para obtener los parámetros del servicio que puedan caracterizar la calidad que recibe el naviero, podemos observar que el tiempo de estancia en puerto de un buque $t_{P}$ es:

... donde:

$$
t_{P}=t_{E}+t_{M}+t_{S}
$$

- $t_{E}$ es el tiempo de espera a ser atendido porque no tiene espacio en el atraque (debido a la congestión portuaria),

- $t_{M}$ es el tiempo de maniobras (llegada de práctico y maniobras de entrada)

- $t_{S}$ es tiempo de servicio en el atraque

En algunos puertos, la existencia de marea podría añadir tiempos adicionales.

Dado que cada buque viene a realizar un movimiento de carga diferente, podemos entender que el naviero aspire a obtener lo que definiríamos como una alta productividad de la escala. Esta productividad $P_{P}$ se puede formular como:

$$
P_{P}=\frac{q}{t_{P}}=\frac{q}{t_{S}+t_{M}+t_{E}}
$$

Despreciando $t_{M}$ frente a $t_{S}$ obtenemos:

$$
P_{P}=P \cdot\left(\frac{1}{1+\frac{t_{E}}{t_{S}}}\right)
$$

A la relación $\frac{t_{E}}{t_{S}}$ se la denomina espera relativa $\epsilon$. La productividad de la escala $P_{P}$ tiene pues 2 términos:

- Término de productividad del buque atracado: $P$

- Término de influencia de la congestión: medido con el estimador $\epsilon$, que repercute en el cuantificador en términos de $\frac{1}{1+\epsilon}$

Esta expresión se puede verbalizar diciendo que el naviero desea una alta productividad en el atraque, $P$, y que la congestión no afecte en exceso a este valor, dando como resultados 
una productividad de la escala $P_{P}$ muy diferente.

Esta sencilla ecuación nos muestra la importancia de considerar la congestión, a la hora de valorar la calidad del servicio que recibe el naviero. Y nos muestra además, que el estimador más adecuado para caracterizarla es precisamente la espera relativa $\epsilon$.

Para determinar la Capacidad deberemos pues relacionar $\rho_{\text {lim }} \operatorname{con} \epsilon$, fijando un $\epsilon_{\text {lim }}$. A la relación $\{\rho-\varepsilon\}$ la denominamos curva de la congestión. En la literatura científica Agerschou (2004)- se suele encontrar que el valor propuesto más frecuente para $\epsilon_{\text {lim }}$ es 0,1 . Aunque en algunos otros se ofrecen rangos de valores mayores.

\section{EL APOYO EN LA TEORÍA DE COLAS}

A la hora de estudiar la relación $\{\rho-\varepsilon\}$, es recurrente acudir al auxilio de la teoría de colas, que desarrollada en otros campos de la técnica pronto se aplicó en el campo de la ingeniería portuaria. Para ello se precisa caracterizar las llegadas de los buques (en la que existe una amplia unanimidad en que se consideren un suceso Poisson, con lo que el tiempo entre dos llegadas de buques consecutivas se distribuye según una función exponencial), y los tiempos de servicio (que suelen ser bien caracterizados por una distribución de Erlang, de parámetro $K$ a definir en cada caso).

De esta manera el muelle queda caracterizado como un sistema de espera, que con la notación de Kendall se expresaría $N / \operatorname{Expon}\{\lambda\} / \operatorname{Erlang}\{\mu, K\}$. Finalmente para sistemas de este tipo, la relación $\{\rho-\varepsilon\}$ tiene un aspecto como el de la figura siguiente.

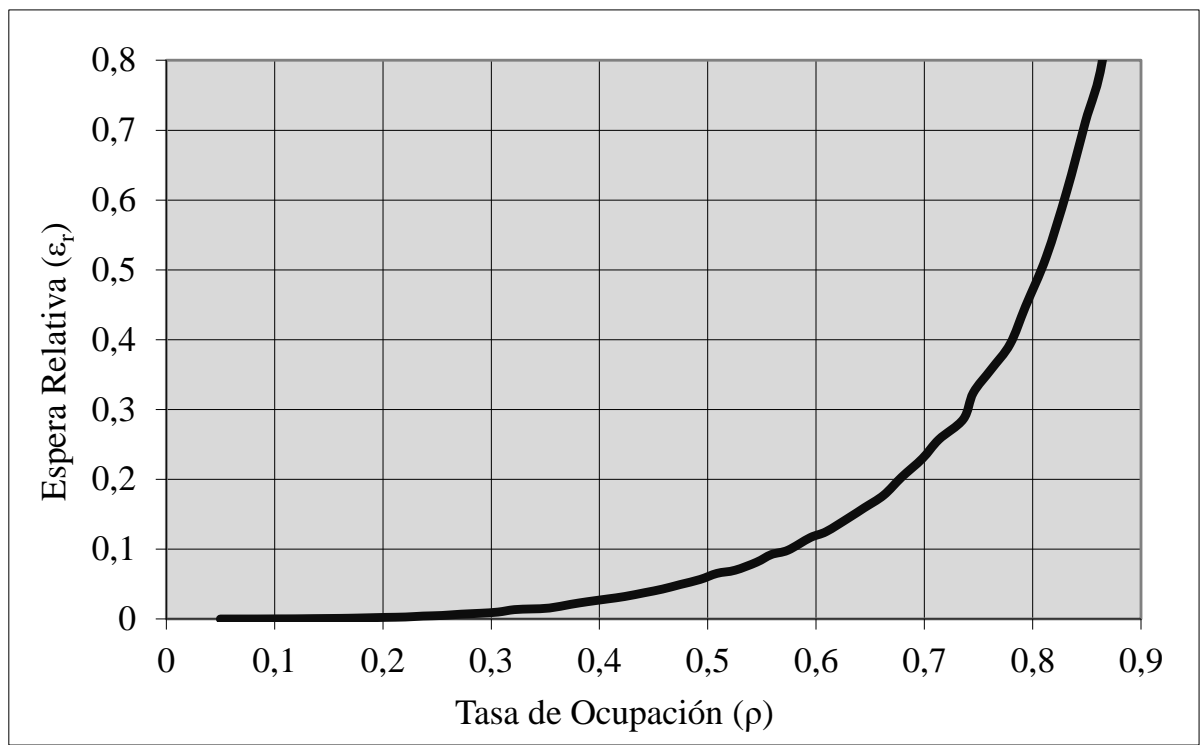

Fig. 1 - Curvar característica de la congestión 


\section{LAS LIMITACIONES DE ESTA MODELACIÓN}

Pero las limitaciones de la teoría de colas son elevadas. La principal proviene de que, en la realidad, la mayoría de los muelles portuarios, y la totalidad de los de contenedores, se gestionan de modo continuo, no identificando los atraques uno a uno. Obrer y Aguilar (2011) y Aguilar y Roser (2013) ya han advertido del error que se comete al pensar que un sistema que se gestiona de modo continuo proporciona esperas similares que los que se gestionan de forma discreta. En el caso continuo hay que caracterizar la variabilidad de las esloras de los buques que llegan, que en la actualidad es muy alta (de 1 a 3), cuando hace apenas 30 años no pasaban de 1 a 1,5 o 1 a 2. Pero también se abre así una nueva vía de optimización que es la asignación de atraque al buque que llega, de entre los huecos que en cada momento existen disponibles en el muelle. Ambos asuntos pueden ser efectivamente simulados mediante programas de simulación; y cuando se ha hecho, se desvela la menor capacidad que se obtiene al compararla con la que la teoría de colas nos daría para los valores medios de los parámetros.

Este asunto también pone en discusión la conveniencia de emplear $N, \rho$ y $P$. Sustituyéndolos por sus valores por metro lineal: L (longitud total del muelle), $\rho_{L}$ (tasa de ocupación, calculada a partir de la ocupación real del muelle, buque a buque) y $P_{L}$ (productividad por metro lineal de buque), como Obrer R. (2016) expone.

Pero todo no queda en esa limitación, pues a menudo nos encontramos con situaciones más difíciles aún de resolver. Por ejemplo, cuando nuestra terminal dispone de 2 alineaciones, situación en la que la variabilidad de las esloras ya anuncia un mayor desaprovechamiento de la terminal que si tenemos 1 sola. O bien cuando tenemos tramos de muelles con calados diferentes, en los que el problema de asignación de atraques a los buques mayores se restringe notablemente.

Como conclusión inicial es que la modelación de la terminal como un simple sistema de espera es defectuosa, sobreestimando la capacidad, lo que se traduce en que los episodios de congestión se producirán antes de lo previsto con los modelos simples.

\section{CONVENIENCIA DE OBTENER DATOS REALES}

Todo lo anterior nos lleva a plantear una pregunta: ¿por qué tenemos que modelar a priori la terminal? U otra aún peor: ¿por qué no podemos comprobar los valores que una modelación, a menudo tan simple, produce? La respuesta es muy sencilla: porque no se toman datos reales.

Para la obtención de datos reales de explotación se precisa:

- Una Autoridad Portuaria interesada en monitorizar el nivel de servicio de sus terminales, es decir que asuma estratégicamente la necesidad de tomar datos.

- Que la medición de los datos sea sistemática y fiable, de calidad. Lo ideal es que la medición se integrara en alguno de los sistemas de garantía de calidad de la institución. Y que los datos se analicen periódicamente, por ejemplo trimestralmente, detectando y corrigiendo los problemas de calidad de la información adquirida.

- Que todo dato a medir sea motivo de una cuidadosa forma de definir cómo se adquiere el dato. A este fin sería conveniente algún acuerdo a nivel de sistema portuario, nacional (interviniendo Puertos del Estado), o internacional (ESPO u otros). 
- Que esté perfectamente claro quién es el responsable de tomar las medidas, y quién de almacenarlas y custodiarlas.

No es necesario medir solamente en momentos de congestión, sino sistemáticamente, día a día, buque a buque. Incluso con situaciones de tráfico alejadas de los límites, se producen las situaciones de congestión. Estos episodios son los que conforman la aparición de colas. Lo que ocurre no es que en situaciones alejadas de los límites no aparezcan estos episodios, sino que son menos probables. Conociendo cómo se comporta la terminal en estos episodios podremos extrapolar hacia situaciones en las que estos episodios sean más probables. Podremos así barrer el espacio $\{\rho-\varepsilon\}$ o bien la $\left\{\rho_{L}-\varepsilon\right\}$ obteniendo a partir de datos reales la curva característica de la congestión de la terminal. Y obtener con ello la evolución de los niveles de servicio de la terminal (Aguilar, J. y Obrer, R. 2012).

Además, podremos plantear modelaciones numéricas de la terminal y comprobar sus resultados. De forma que no ocurra como ahora pasa, que la comprobación lo es entre modelaciones sin contraste con realidad. Se podrán ensayar así los efectos de la optimización de la asignación de atraques, o los de adopción de políticas de prioridad, u otros, con más rigor.

Por último alguna observación sobre los datos a tomar:

- Sobre la llegadas de buques. A veces se objeta que cuando la terminal está congestionada los buques disminuyen su velocidad provocando esperas inferiores a las que se producirían de no conocer este estado. Este dato hay que tenerlo en cuenta, pues redunda en un peor servicio del esperado. Por ello, una vez fijado el punto a partir del cual se considera que el buque ha llegado a puerto, se puede corregir este valor con los datos de la posición que el buque ha tenido en las últimas horas, a partir de la información que actualmente entregan sistemas como el AIS. Esta información puede empezar a obtenerse 1 o 2 días antes de la llegada del buque, a partir de la ETA de la que dispongamos.

- Ocupación del atraque. El atraque se considera ocupado cuando no puede ser asignado a otro. Por ello cuando el práctico reciba la orden de acudir a bordo, siendo ya conocedor del destino de la embarcación, puede considerarse que su espacio asignado ya está ocupado. El espacio a asignar dependerá los cruces de amarras que el servicio de amarre prevé en el puerto, considerando también si los atraques se atribuyen a múltiplos de noray o no. Así mismo, dejará de estar ocupado cuando el práctico reciba la orden de subir a bordo para realizar la maniobra de salida.

- Esperas, serán las que se produzcan ente el momento de llegada y el de asignación del atraque antes indicado.

- Movimientos realizados, al menos los de contenedores a embarcar y desembarcar, aunque mejor completar, si la terminal lo facilita, con las remociones y otros movimientos complementarios como los de las tapas de escotillas que se hayan practicado.

Los valores medio anuales, o incluso trimestrales, de la posición de la terminal en el espacio $\{\rho-\varepsilon\}$ nos permiten tener controlada la terminal en término de niveles de servicio, con la consiguiente estimación de la tendencia hacia niveles de servicio futuros, de manera que puedan proponerse a tiempo correcciones fundadas, como mejoras en la productividad (grúas), que restituyan la posición de la terminal en este espacio. 


\section{CONCLUSIONES}

En esta ponencia se han desarrollado de forma rápida las bases conceptuales del cálculo de la capacidad de terminales portuarias de contenedores. Se ha constatado la dependencia que estos modelos tienen de ciertos parámetros que se obtienen a partir de la teoría de colas.

Se han visto los defectos que esta simplificación tiene. Se ha enunciado cómo incluso los modelos de simulación se muestran insuficientes a menudo, tanto por la simplificación que conllevan como por la falta de contraste.

Se concluye acerca de la necesidad de que las autoridades portuarias realicen medidas concernientes a la caracterización del espacio de medida de la congestión $\{\rho-\varepsilon\}$ de todas sus terminales de contenedores, como forma de tener controlada la evolución de cada una de ellas a lo largo del tiempo.

\section{REFERENCIAS}

AGERSCHOU, H. (2004). Planning and design of ports and marine terminals. Thomas Telford Ltd, London.

AGUILAR, J. y OBRER, R. (2012). Una propuesta para la definición de los niveles de servicio medios del atraque en terminales portuarias. CIT 2012. Granada, 20 a 22 de junio de 2012.

AGUILAR, J. y OBRER, R. (2013). Método probabilístico para determinar la longitud de un atraque calculado con base en la explotación de atraques discretos que atienden flotas con variabilidad de esloras. XII Jornadas de Costas y Puertos. Cartagena, 7 y 8 de mayo de 2013. DE MONIE, G. (1988). Medición y evaluación del rendimiento y de la productividad de los puertos. Monografías de la UNCTAD sobre gestión de los puertos. New York.

MONFORT, A.; AGUILAR, J.; VIEIRA, P.; MONTERDE, N.; OBRER, R.; CALDUCH, D.; MARTÍN, A. M.; y SAPIÑA, R. (2011). Manual de capacidad portuaria: aplicación a terminales de contenedores. Fundación Valenciaport, Valencia.

OBRER, R. y AGUILAR, J. (2011). A influencia da variabilidade dos comprimentos dos navios no cálculo de capacidade dos cais de containers. 7as Jornadas portuguesas de engenheria costeira e portuaria. Oporto, 6 y 7 de octubre de 2011.

OBRER, R. (2016). Nueva determinación de la capacidad de muelles en terminales de contenedores mediante técnicas de simulación. Tesis doctoral. Universitat Politècnica de València. Valencia.

RODRÍGUEZ, F. (1977). Capacidad de los muelles. MOPT, Madrid. 